\title{
Measurement of the Average Mass of Proteins Adsorbed to a Nanoparticle by Using a Suspended Microchannel Resonator
}

\author{
M. REZA NEJADNIK, WIM JISKOOT \\ Division of Drug Delivery Technology, Leiden Academic Centre for Drug Research (LACDR), Leiden University, Leiden 2333 CC, \\ The Netherlands
}

Received 31 July 2014; revised 16 September 2014; accepted 19 September 2014

Published online 15 October 2014 in Wiley Online Library (wileyonlinelibrary.com). DOI 10.1002/jps.24206

\begin{abstract}
We assessed the potential of a suspended microchannel resonator (SMR) to measure the adsorption of proteins to nanoparticles. Standard polystyrene beads suspended in buffer were weighed by a SMR system. Particle suspensions were mixed with solutions of bovine serum albumin (BSA) or monoclonal human antibody (IgG), incubated at room temperature for $3 \mathrm{~h}$ and weighed again with SMR. The difference in buoyant mass of the bare and protein-coated polystyrene beads was calculated into real mass of adsorbed proteins. The average surface area occupied per protein molecule was calculated, assuming a monolayer of adsorbed protein. In parallel, dynamic light scattering (DLS), nanoparticle tracking analysis (NTA), and zeta potential measurements were performed. SMR revealed a statistically significant increase in the mass of beads because of adsorption of proteins (for BSA and IgG), whereas DLS and NTA did not show a difference between the size of bare and protein-coated beads. The change in the zeta potential of the beads was also measurable. The surface area occupied per protein molecule was in line with their known size. Presented results show that SMR can be used to measure the mass of adsorbed protein to nanoparticles with a high precision in the presence of free protein. (C) 2014 Wiley Periodicals, Inc. and the American Pharmacists Association J Pharm Sci 104:698-704, 2015
\end{abstract}

Keywords: adsorption; aggregation; albumin; nanoparticles; particle sizing; proteins; resonance mass measurement; analytical chemistry

\section{INTRODUCTION}

The use of nanoparticles for biomedical applications has been a major area of study in the last decade and continues to be a rapidly growing field of research. Nanoparticles are rigorously researched in the field of drug delivery and imaging not only because of their size, shape, or high surface to volume ratio, but also because they are looked at as platforms that offer versatile possibilities for modification with functional moieties ranging from small chemical groups to large macromolecules. ${ }^{1}$ Drugs, proteins, fluorescent dyes, and targeting ligands are examples of functional moieties that are attached to nanoparticles for specific functions. In addition, the surface of nanoparticles is often coated with molecules such as polyethylene glycol (PEG) to stabilize the particle suspension or reduce its unspecific interaction with proteins and other components of the biological environment. ${ }^{1,2}$

The abovementioned moieties are generally immobilized on the surface of nanoparticles either through a covalent linkage or by a simple adsorption process. Regardless of the method of immobilization, it is a challenge to quantify the amount of immobilized moiety on the surface of a nanoparticle. ${ }^{3,4}$ Most of the used quantification methods are based on measurement of the depletion of the component of interest in the solution that is used to coat the nanoparticles. ${ }^{5}$ These so-called indirect methods have several drawbacks, including: (1) they do not provide a proof that the moiety is actually coated on the nanoparticles, as it could well be adsorbed to other interfaces encountered in the adsorption or sample preparation process; (2) they are susceptible to the presence of unbound impurities or contaminants;

Correspondence to: Wim Jiskoot (Telephone: +31-71-527-4570; Fax: +31-71527-4565; E-mail: w.jiskoot@lacdr.leidenuniv.nl)

Journal of Pharmaceutical Sciences, Vol. 104, 698-704 (2015)

(C) 2014 Wiley Periodicals, Inc. and the American Pharmacists Association
(3) they are inaccurate when only a small fraction of the moiety is coated; and (4) the overall quantification requires knowing the exact number of nanoparticles coated in the process. There are also methods that aim for direct measurement of the amount of immobilized components. The majority of such methods are, however, only suitable for qualitative or semiquantitative assessments and do not provide precise indicates of the amount of immobilized components. For instance, fluorescent labeling of proteins has been frequently used to evaluate the adsorption of proteins to the surface of gold nanoparticles. ${ }^{5}$ Other than being nonquantitative, such methods employ modified versions of the immobilized component (e.g., fluorescently labeled protein) that may influence the immobilization behavior and lead to inaccurate indications. ${ }^{3}$ Generally used sizing methods such as dynamic light scattering (DLS) and nanoparticle tracking analysis (NTA) have also been used to evaluate the immobilized components on the surface of nanoparticles. . $^{3,6,7}$ These methods provide information regarding the hydrodynamic thickness of the adsorbed layer and not directly about the adsorbed amount. Several other techniques such as X-ray photoelectron spectroscopy or zeta potential measurements can offer proof on the presence of a coating on the nanoparticles without providing quantitative data. Scanning and transmission electron microscopy and atomic force microscopy may also be useful for evaluation of the coatings; however, these methods have an extremely low throughput and often require interfering treatments (such as drying, freezing, or deposition of a conductive layer) before an image can be made..$^{3,4,8}$

Recently developed suspended microchannel resonators (SMR) enable the measurement of the mass of single nanoparticles with precision in the range of femtogram to attogram. ${ }^{9-11}$ In these systems, a suspension of particles is flushed through a microchannel inside the resonator. The resonance frequency of the suspended microchannel is highly sensitive to the 
presence of particles whose density differs from that of the flushed fluid. Passage of a particle with a density higher than that of the fluid will cause a decrease in the resonance frequency of the microchannel. This decrease in frequency is proportional to the buoyant mass of the particle in the surrounding fluid. SMR systems have been previously used for studying the mass of various micro- and nanoparticles including protein particles and also for quantitative differentiation of protein particles and silicone oil droplets. ${ }^{11,12}$

The aim of this study was to evaluate the potential of the SMR, also referred to as resonant mass measurement, ${ }^{12}$ for measuring the mass of proteins adsorbed to nanoparticles. To this end, proteins in different formulations were allowed to adsorb to model polystyrene beads and the resulting coated beads were weighted by using SMR. For comparison, DLS, NTA, zeta potential, and BCA protein assay measurements were also performed. Our data show that SMR provides a precise estimate of the average mass of adsorbed protein per nanoparticle without the need for separation from unadsorbed protein.

\section{MATERIALS AND METHODS}

\section{Materials}

Polystyrene standard beads with diameters of 600 and $1000 \mathrm{~nm}$ were purchased from Fisher Scientific (Landsmeer, The Netherlands). Monoclonal human antibody of the IgG1 subclass (IgG) was kindly provided by Boehringer Ingelheim (Biberach, Germany). Bovine serum albumin (BSA) and other chemicals were purchased from Sigma-Aldrich (Steinheim, Germany). Ultrapure water (18.2 $\mathrm{M} \Omega \mathrm{cm}$ water) was dispensed by using a PURELAB Ultra water purification system (ELGA LabWater, Ede, The Netherlands).

\section{Adsorption Experiments}

The polystyrene bead suspensions (diameter of 600 and $1000 \mathrm{~nm}$ ) were diluted $2000 \times$ or $1000 \times$, respectively, in $10 \mathrm{mM}$ phosphate buffer (PB), $\mathrm{pH} 7.0$ to reach a concentration that was optimal for measurements, that is, about $10^{7}$ beads $/ \mathrm{mL}$. BSA solutions $(1,0.1$, and $0.01 \mathrm{mg} / \mathrm{mL})$ in $\mathrm{PB}$ were prepared. IgG solution $(0.1 \mathrm{mg} / \mathrm{mL})$ in $\mathrm{PB}$ was also prepared. Two milliliter of the bead suspension was mixed with $2 \mathrm{~mL}$ of the protein solution and the mixture was incubated for $3 \mathrm{~h}$ (long enough to reach saturation of the surface, as indicated by preliminary experiments) at room temperature after which the measurements were performed.

\section{SMR Measurements}

Suspended microchannel resonator measurements were performed using an Archimedes system (Malvern Instruments, Malvern, UK). A microsensor chip with internal microchannel dimensions of $8 \times 8 \mu \mathrm{m}^{2}$ was used for all experiments. Prior to experiments, the sensor was calibrated with $1.034 \mu \mathrm{m}$ polystyrene size standards as instructed by the manufacturer. The sensor was rinsed with $2 \%$ sodium dodecyl sulfate solution and washed thoroughly with several runs of ultrapure water before each measurement. The sample was loaded to the sensor for $30 \mathrm{~s}$. The limit of detection was fixed to $0.018 \mathrm{~Hz}$, which was well above the noise level and found to be optimal for detection of the nanoparticles used herein. The measurement was continued for $10 \mathrm{~min}$ at room temperature, which allowed detection of at least 2000 particles. The buoyant mass of particles before (bare polystyrene beads) and after (BSA-coated and IgGcoated polystyrene beads) adsorption of protein was measured. The distribution of particles with a buoyant mass within the range of 0-40 femtogram was plotted by choosing a bin size of 0.25 femtogram. The average buoyant mass of a certain population of particles was calculated by averaging the mass of particles under the peak associated to that population in the distribution graph. The PB and BSA solution were also tested as control samples. In order to investigate whether the presence of unbound protein would influence the measurement of the mass of particles, two control measurements were conducted: (1) the beads were spun down by using a centrifuge (Microfuge 18; Beckman Coulter Inc., Brea, California) at $10,000 \mathrm{~g}$ for 10 min, resuspended in $\mathrm{PB}$ and weighed again and (2) the mixture was diluted 10-fold with PB and measured again. For each condition, three independent measurements with separately prepared mixtures of polystyrene beads and proteins were performed.

The average buoyant mass of the adsorbed protein layer to a single nanoparticle (i.e., the average change in the buoyant mass of beads) was converted to the real mass of the protein layer according to Eq. 1, assuming the density of the protein to be $1.35 .^{13}$ The densities of different fluids were measured by SMR and were statistically equal to the density of ultrapure water. Therefore, the value of $1 \mathrm{~g} / \mathrm{mL}$ was used as the density of fluid in all calculations.

$$
M=\frac{M_{\mathrm{B}}}{\left(1-\rho_{\text {fluid }} / \rho_{\text {particle }}\right)}
$$

From the calculated average mass of proteins adsorbed to a nanoparticle and the known molar mass of the protein, the average number of protein molecules adsorbed to a single nanoparticle was calculated. Subsequently, the average surface area per each protein molecule was calculated, by assuming a monolayer of protein on the nanoparticle surface.

\section{Dynamic Light Scattering}

Dynamic light scattering measurements were performed with a Malvern Zetasizer Nano ZS (Malvern, Herrenberg, Germany) equipped with a 633-nm He-Ne laser and operating at an angle of $173^{\circ}$. The software used to collect and analyze the data was the Zetasizer Software version 7.03 from Malvern. Fivehundred microliter of each sample was measured in single-use polystyrene half-micro cuvettes (Fisher Emergo, Landsmeer, The Netherlands) having a path-length of $10 \mathrm{~mm}$. The measurements were made at a position of $4.65 \mathrm{~mm}$ from the cuvette wall with an automatic attenuator and at a controlled temperature of $25^{\circ} \mathrm{C}$. For each sample, 10 runs of $15 \mathrm{~s}$ were performed. The Zaverage (Z-ave) diameter, polydispersity index, and peak center of the intensity distribution were obtained from the autocorrelation function by using the "general purpose mode" analysis model. These parameters were calculated from three independent measurements with separately prepared samples.

\section{Zeta Potential Measurements}

The particles' zeta potential was determined by laser Doppler electrophoresis with the same instrument as used for DLS measurements. The average and standard deviation of the zeta potential were calculated from three independent measurements with separately prepared samples. 


\section{Nanoparticle Tracking Analysis}

Nanoparticle tracking analysis measurements were performed with a NanoSight LM20 (NanoSight, Amesbury, U K), equipped with a sample chamber and a 640-nm laser. The samples were injected in the sample chamber with sterile syringes (BD Discardit II, Franklin Lakes, New Jersey). The software used for capturing and analyzing the data was the NTA 2.0 Build 127. The samples were measured for $40 \mathrm{~s}$ with manual shutter and gain adjustments from which the mean diameter of the particles was obtained. These experimental settings resulted in detection of at least 3500 particles per measurement, out of which 100-200 particles could be tracked long enough to render reliable size measurements. The average and standard deviation of the mean diameter were calculated from three independent measurements with separately prepared samples.

\section{Measurement of Protein Concentration in the Supernatant}

The amount of protein in the starting solution and in the supernatant after the coating process was determined by using a microbicinchoninic acid-based (micro-BCA) protein assay kit (Pierce, Rockford, Illinois) according to the manufacturer's instructions. In order to remove the polystyrene beads after adsorption of proteins, a centrifugation step at $10,000 \mathrm{~g}$ for $10 \mathrm{~min}$ was performed that efficiently removed more than $90 \%$ of the beads.

\section{Statistical Analysis}

Statistical calculations were performed with the available online GraphPad Software by using a one-tailed $t$-test, unless otherwise stated. In order to check whether the variances belonging to populations of the bare and BSA-coated polystyrene beads were different, a two tailed F-test (data analysis package of Microsoft Excel) was performed. Differences were considered statistically significant for $p$ values smaller than 0.05 .

\section{RESULTS AND DISCUSSION}

To the best of our knowledge, this is the first study to show that the adsorption of protein to the surface of polystyrene beads leads to a clear increase in the buoyant mass of the beads that is measurable with a SMR system (Fig. 1). As one would expect, the SMR did not detect particles in control samples of only PB or BSA solution, whereas a large number of particles were detected in suspensions of both bare and BSA-coated polystyrene beads. Statistical analysis of the data on the buoyant mass of particles before (3210 beads weighed with an average buoyant mass of $7.49 \pm 0.76$ femtogram) and after BSA adsorption (3609 beads weighed with an average buoyant mass of $8.04 \pm 0.79$ femtogram) revealed that there is a statistically significant difference between the two populations $(p<0.0001)$. F-test analysis of these data suggest that the variance of the buoyant mass of particles in each population (under each peak), which is a measure of the peak broadness and can be used as an indicator of the polydispersity, increased significantly ( $p<$ 0.005) after adsorption of BSA. This observation may suggest that the adsorption of protein slightly increased the polydispersity of the particles in terms of their mass distribution.

The distribution of the particles' buoyant mass remained essentially the same after they were spun down and resuspended in PB and also after dilution with PB (Fig. 2). This observation confirmed that the change in buoyant mass was directly related

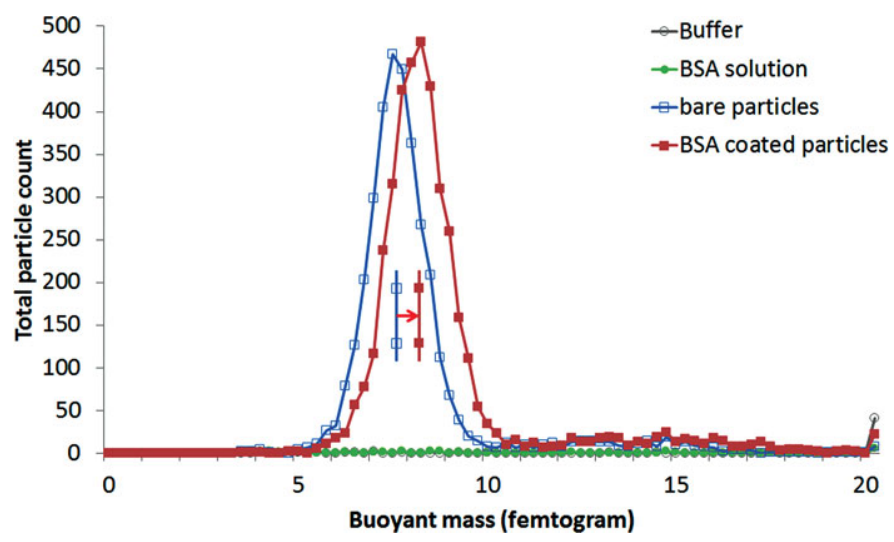

Figure 1. Distribution of the buoyant mass of particles for buffer (empty circles, gray), BSA solution (filled circles, green), suspension of bare $600-\mathrm{nm}$ polystyrene beads (empty squares, blue) and BSA-coated $600-\mathrm{nm}$ polystyrene beads (filled squares, red). The arrow shows the shift in the average buoyant mass of particles under the peak because of adsorption of BSA.

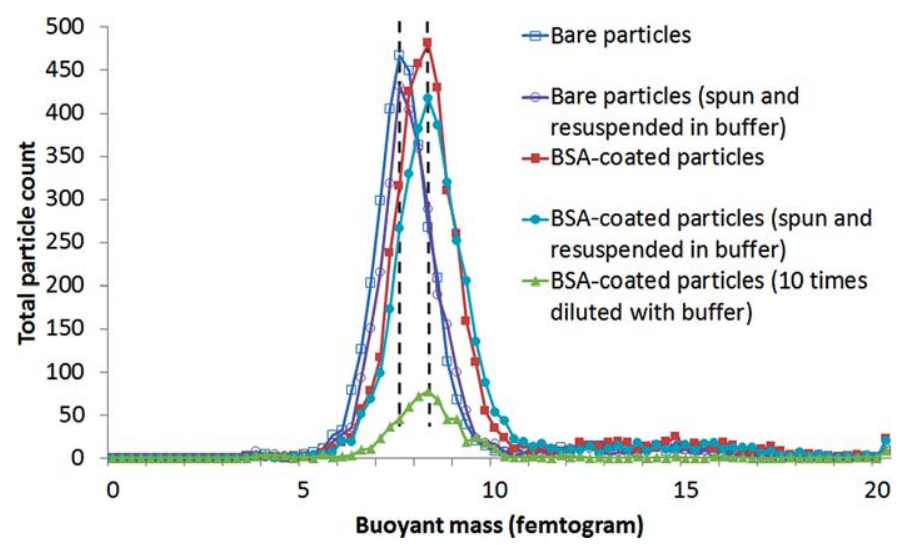

Figure 2. Distribution of the buoyant mass of particles for suspensions of bare 600-nm polystyrene beads (empty squares, blue), bare $600-\mathrm{nm}$ polystyrene beads spun down and resuspended in buffer (empty circles, purple), BSA-coated $600-\mathrm{nm}$ polystyrene beads (filled squares, red), BSA-coated 600-nm polystyrene beads spun down and resuspended in buffer (filled circles, light blue), and BSA-coated 600-nm polystyrene beads 10 times diluted in buffer (filled triangles, green). The dashed lines are eye guides to show that the peaks are the same after removal or dilution of the excess protein in the surrounding fluid.

to the adsorption of BSA and was independent of the presence or absence of the unbound BSA in the surrounding fluid. This observation also indicates that the BSA is irreversibly adsorbed to the polystyrene beads (no desorption upon dilution or resuspension in $\mathrm{PB}$ ). It has to be realized, however, that this conclusion may only be valid for this particular protein at the concentrations used in this study. Different proteins and higher concentration of the protein may lead to the presence of loosely interacting protein around the polystyrene beads that may desorb upon dilution or resuspension in PB.

In order to check the reproducibility of the measurements, three independent measurements with separately prepared samples were performed. Table 1 summarizes the experimental results on the average buoyant mass (as measured by SMR), the size (as measured by DLS and NTA), and the zeta potential (as measured by laser Doppler electrophoresis) for both bare and 
Table 1. Summary of the Experimental Results on the Buoyant Mass of the 600-nm Polystyrene Beads (as Measured by SMR), the Particle Size (Z-Ave and Peak Center of the Intensity Distribution as Measured by DLS and Mean Diameter as Measured by NTA), and the Zeta Potential of Particles (as Measured by Laser Doppler Electrophoresis) for Both Bare and Protein-Coated Beads

\begin{tabular}{lcrrr}
\hline & Bare Particles & Protein-Coated Particles & Change & $p$ Value \\
\hline Average buoyant mass of particles (femtogram) & $7.45 \pm 0.07$ & $8.00 \pm 0.03$ & $0.55 \pm 0.10$ & $<0.0001$ \\
Zeta potential (mV) & $-72.5 \pm 7.9$ & $-35.5 \pm 3.3$ & $37.0 \pm 5.2$ & 0.010 \\
Z-ave (nm) & $618.8 \pm 16.1$ & $621.2 \pm 3.6$ & $2.4 \pm 17.4$ & 0.407 \\
Peak center of the intensity distribution as measured by DLS (nm) & $637.0 \pm 3.4$ & $653.0 \pm 9.6$ & $16.0 \pm 12.3$ & 0.026 \\
Mean diameter from NTA (nm) & $623.3 \pm 68.6$ & $602.2 \pm 17.7$ & $-21.2 \pm 50.9$ & 0.314 \\
\hline
\end{tabular}

The data represent the average and standard deviations for three independent measurements with separately prepared samples. The results of the statistical analyses are also summarized.
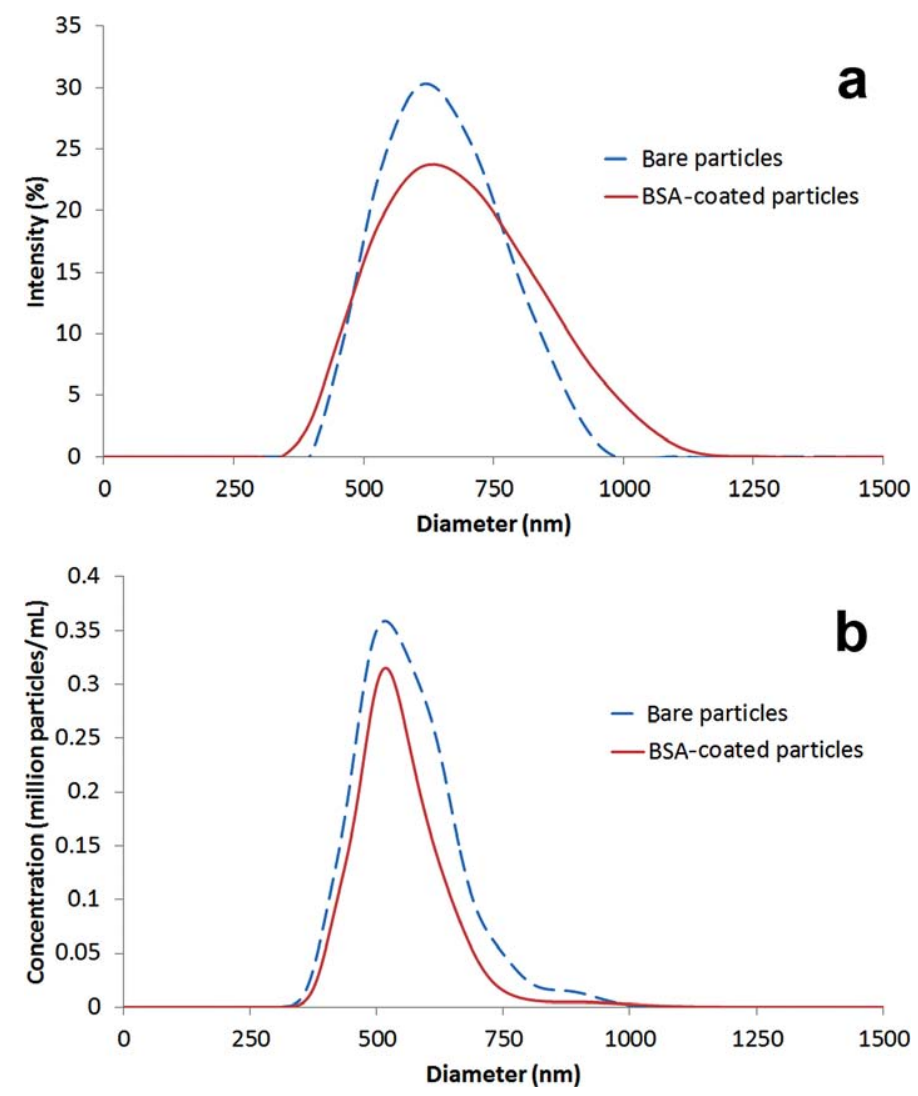

Figure 3. Size distribution of particles for suspensions of bare $600-\mathrm{nm}$ polystyrene beads (dashed line, blue), and BSA-coated 600-nm polystyrene beads (solid line, red) as obtained by (a) DLS and (b) NTA.

protein-coated polystyrene beads. The representative size distributions obtained by DLS and NTA are presented in Figure 3. The SMR method showed a highly significant difference between the buoyant mass of bare and protein-coated polystyrene beads. Similarly, zeta potential measurements showed a statistically significant difference between the bare and BSA-coated polystyrene beads, confirming the presence of the protein coating. These results are in agreement with the literature, as adsorption of considerable amounts of BSA to polystyrene surfaces with different charges has been reported. ${ }^{14,15}$

In contrast, the sizing methods, that is, DLS and NTA, did not show any statistically significant differences between the $\mathrm{Z}$-ave and the mean diameter of the bare and protein-coated polystyrene beads ( $p>0.05$; see Table 1$)$. The peak center of the intensity distribution as measured by DLS did reveal an increase in the size of particles because of adsorption of protein that was statistically significant. Nevertheless, these data show the limitations of DLS and NTA, in terms of precision, for detection of small changes in diameter of $600-\mathrm{nm}$ beads because of adsorption of a protein layer. Moreover, DLS and NTA are not suitable for assessing the amount of adsorbed protein. It is, however, noteworthy that these methods have been used to detect a hydrated coating for small particles (diameter of 20-200 $\mathrm{nm}$ ) and for relatively thick PEG coatings (about $30 \mathrm{~nm}$ thick). 6,7,15 The micro-BCA assay was also not sensitive enough to detect a reduction in the concentration of BSA because of adsorption to polystyrene beads (Table 2). It has to be realized that the amount of protein adsorbed in these experiments may have been too small in comparison to the detection limit of the micro-BCA assay and the protein concentration of the starting solution $(0.05 \mathrm{mg} / \mathrm{mL})$, making it difficult to detect any changes with such protein assays. Altogether, the abovementioned data clearly show the limitation of the conventional techniques and emphasize the power of SMR in quantification of the amount of protein adsorbed to single nanoparticles.

The average mass of the adsorbed BSA to a polystyrene bead was calculated to be $2.1 \pm 0.4$ femtogram, which is equal to the mass of 19,062 \pm 3679 BSA molecules (molar mass of BSA is $66.5 \mathrm{kDa}$ ). Assuming a smooth surface for a polystyrene bead with a diameter of $600 \mathrm{~nm}$ and a protein monolayer, the average surface area per BSA molecule was calculated to be $59.3 \pm 11.4 \mathrm{~nm}^{2}$. At the same time, theoretical calculations

Table 2. Concentration of BSA in the Starting Solution and in the Supernatant After the Coating Process, as Measured by Micro-BCA Assay

BSA in Supernatant After

Adsorption of Protein to

BSA in Starting Solution 600-nm Nanoparticles

Change $\quad p$ Value

$\begin{array}{lllll}\text { Concentration as measured by } & 0.048 \pm 0.001 & 0.047 \pm 0.001 & -0.001 \pm 0.001 & 0.078\end{array}$

BCA assay $(\mathrm{mg} / \mathrm{mL})$

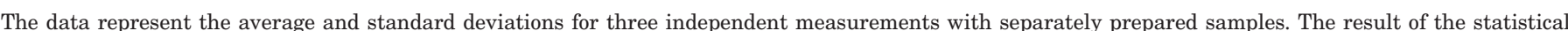
analysis is also presented. 
Table 3. Summary of the Experimental Results on the Buoyant Mass of the 1000-nm Polystyrene Beads for Both Bare and Protein-Coated Beads as Measured by SMR

\begin{tabular}{lcrrr}
\hline & Bare Particles & Protein-Coated Particles & Change & $p$ Value \\
\hline Average buoyant mass of particles (femtogram) & $33.30 \pm 0.07$ & $34.50 \pm 0.25$ & $1.17 \pm 0.31$ & 0.001 \\
\hline
\end{tabular}

The data represent the average and standard deviations for three independent measurements with separately prepared samples. The result of the statistical analysis is also presented.

suggest that the projected area of an adsorbed BSA molecule with a hydrodynamic diameter of $6.5 \mathrm{~nm}^{16}$ would be $33.2 \mathrm{~nm}^{2}$. This value translates in an average area of 36.9 and $60.7 \mathrm{~nm}^{2}$ for hexagonal close packing (90.7\% surface coverage $\left.{ }^{17}\right)$ and random sequential adsorption (54.7\% surface coverage $\left.{ }^{18,19}\right)$ models, respectively. In particular, the value for the random sequential adsorption model is in excellent agreement with our experimental findings.

Suspended microchannel resonator measurements showed a buoyant mass of $33.30 \pm 0.07$ femtogram for bare polystyrene beads with a diameter of $1000 \mathrm{~nm}$ (Table 3), which is approximately 4.5 times the buoyant mass of the $600-\mathrm{nm}$ beads. This ratio is proportional to the ratio between the volumes of these particles (4.6) that have the same density $(1.05 \mathrm{~g} / \mathrm{mL})$. This agreement in the ratios revealed that SMR is a reliable method in measurement of the relative masses of particles with different sizes. The average surface area per each BSA molecule on 1000 -nm beads $\left(76.8 \pm 20.5 \mathrm{~nm}^{2}\right)$ was not different from that for $600-n m$ beads. Such similarity was expected because the two particles are of the same type and should have similar surface properties.

In order to further validate the SMR measurement of the mass of protein adsorbed to nanoparticles, adsorption from BSA solutions of different concentration $(0.5,0.05$, and $0.005 \mathrm{mg} / \mathrm{mL}$ ) to $600-\mathrm{nm}$ polystyrene beads was studied. Figure 4 shows the average adsorbed mass of BSA on a nanoparticle as well as the associated mean surface area per BSA molecule for three BSA concentrations. The adsorbed mass of BSA per particle decreased with decreasing the concentration of the protein. Consequently, this decrease was accompanied with an increase in the calculated surface area per BSA molecule. Considering that the surface was saturated by protein for all concentrations, these results can be interpreted by the relative

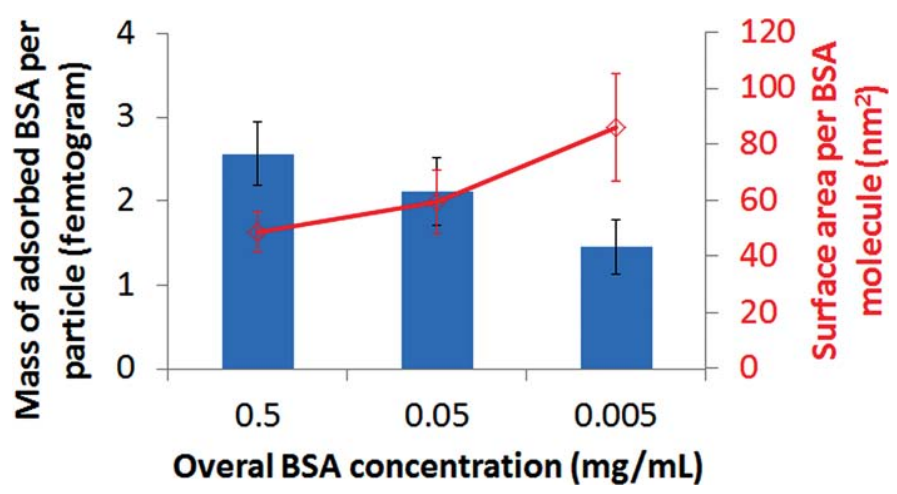

Figure 4. Adsorbed mass of BSA on a 600-nm polystyrene bead (left axis, blue bars) and the associated surface area per BSA molecule (right axis, red-filled squares) for BSA concentrations of $0.5,0.05$, and $0.005 \mathrm{mg} / \mathrm{mL}$. The error bars represent the standard deviations calculated from three separate measurements. rates of supply and spreading of the protein. When the supply rate of protein is low (from low-concentration BSA solution), the adsorbed BSA molecules have the time to spread on the surface (to maximize favorable bounds with the substrate) before sensing a neighboring molecule. As a result, a larger area is occupied per BSA and less protein can be adsorbed per unit area of the surface. On the contrary, when using a high concentration of BSA, a supply rate that is higher than the spreading rate can lead to a rapid crowding of the surface of polystyrene beads. This crowding would, in turn, force the protein to keep a more compact structure because the spreading is sterically inhibited. ${ }^{20-22}$ These results are in line with reported observations for adsorption of $\alpha$-lactalbumin on silica at different protein concentrations where the spreading rate was in the order of $100 \mathrm{~s}$ and the surface was coated with protein over the time course of 7.5-750 s depending on the concentration. ${ }^{22}$

The applicability of the method to other proteins with different characteristics (e.g., net charge of the protein molecule) was investigated by studying the mass of adsorbed IgG (from a solution with a concentration of $0.05 \mathrm{mg} / \mathrm{mL}$ ) to $600-\mathrm{nm}$ polystyrene beads. Figure 5 shows the distribution of the buoyant mass for bare and IgG-coated polystyrene beads. The average buoyant mass of the particles for each population (shown by separate peaks) is also shown in the graph. The average buoyant mass of particles increased significantly $(p<0.0001)$ from $7.45 \pm$ 0.07 femtogram (bare polystyrene beads) to $8.74 \pm 0.01$ femtogram (IgG-coated beads) because of adsorption of IgG. This increase corresponds to adsorption of $19,899 \pm 1261 \mathrm{IgG}$ molecules and a $56.81 \pm 3.60 \mathrm{~nm}^{2}$ per IgG molecule. This area

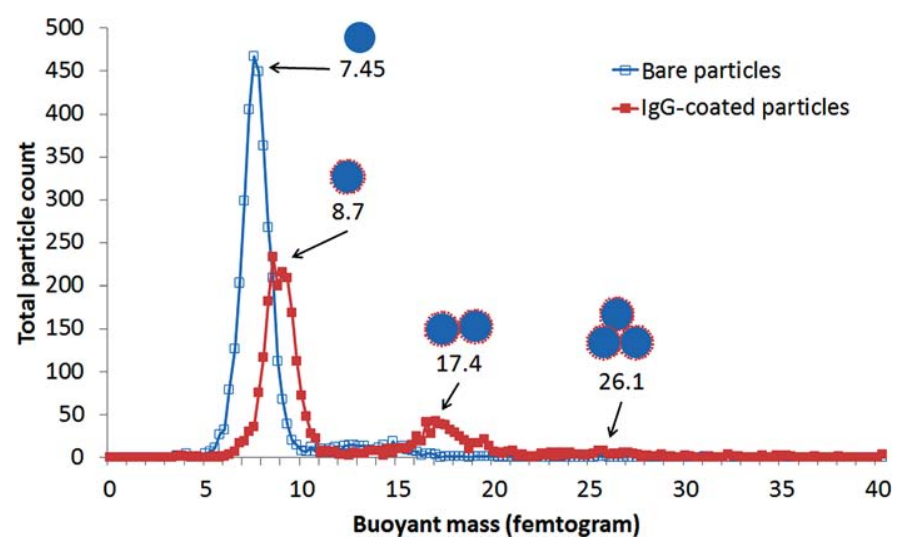

Figure 5. Distribution of the buoyant mass of 600-nm polystyrene beads for suspension of bare (empty squares, blue) and IgG-coated polystyrene beads (filled squares, red). The inset arrows point the detected populations and the associated values are the average buoyant mass of nanoparticles in those populations (under the peaks). The illustrations point out that the second and third peak in the distribution of the IgG-coated particles are related to dimers and trimers of IgG-coated polystyrene beads. 
is in the expected range as the dimensions of the IgG molecule are estimated to be $14 \times 8.5 \times 4 \mathrm{~nm}^{3}{ }^{23,24}$ The zeta potential of the polystyrene beads increased from $-72.5 \pm 7.9$ to $-6.6 \pm$ $0.2 \mathrm{mV}$ (statistically significant difference, $p<0.0001$ ). Clearly, the IgG coating causes a larger increase in the zeta potential as compared with the BSA coating, which is likely related to the difference in the net charge of these molecules (isoelectric point of $\mathrm{BSA} \approx 4.6$ and that of $\mathrm{IgG} \approx 8.5$ ).

Interestingly, two other populations were detected in case of IgG-coated polystyrene beads (Fig. 5). These populations correspond to average buoyant masses of 17.4 and 26.1 femtogram, which are two and three times the buoyant mass of single IgGcoated polystyrene beads. This observation indicates that mixing the bead suspension with IgG solution may have led to the formation of IgG-coated dimer and trimer particles. Considering the positive charge of IgG and the negative charge of bare polystyrene beads, charge-charge interactions are probably responsible for this with the IgG molecules forming a bridge between the polystyrene beads. It has to be emphasized that because SMR measures the mass of particles, it provides an extremely powerful tool for detection and characterization of particle aggregation phenomena.

\section{CONCLUSIONS AND PERSPECTIVES}

The SMR method can be used to measure the average mass of adsorbed protein to a nanoparticle with a high precision. Quantitative mass measurements allow for good estimation of the number of adsorbed protein molecules per nanoparticle and therewith the surface area occupied per protein molecule. Moreover, the SMR method also allows for highly consistent relative mass measurements, making it suitable for studying the aggregation of nanoparticles at high resolution. Other advantages of the method include the direct measurement principle, the possibility to measure minute fractions of adsorbed protein in the presence of a large excess of unbound protein, and a reasonably high sample throughput.

These findings reveal the great potential of SMR methods in characterization of the interaction of nanoparticles with macromolecules and nanoparticles with nanoparticles in the field of pharmaceutical sciences. For instance, a major area of application is the characterization of a protein corona that may be formed around the particles upon contact with biological fluids. ${ }^{25,26}$ The significance of the issue becomes apparent when one realizes that the formation of a corona can alter the biological identity of the nanoparticles and cause them to lose their targeting capabilities. ${ }^{27,28}$ Another area of application would be the aggregation of the pharmaceutical nanoparticles in the presence of secondary moieties such as proteins, which is particularly important because aggregates can influence the fate of the particle-based, biological interventions. ${ }^{29,30}$ Last but not least, nanoparticles with several pharmaceutically relevant surface chemistries could be used as tools to study the adsorption behavior of proteins to surfaces by SMR, for example, as function of protein concentration and formulation composition.

\section{ACKNOWLEDGMENTS}

The research leading to these results has received support from the Innovative Medicines Initiative Joint Undertaking under grant agreement number 115363, resources of which are com- posed of financial contribution from the European Union's Seventh Framework Programme (FP7/2007-2013) and EFPIA companies' kind contribution.

\section{REFERENCES}

1. Doane TL, Burda C. 2012. The unique role of nanoparticles in nanomedicine: Imaging, drug delivery and therapy. Chem Soc Rev 41(7):2885-2911.

2. Petros RA, DeSimone JM. 2010. Strategies in the design of nanoparticles for therapeutic applications. Nat Rev Drug Discov 9(8):615-627. 3. Mahmoudi M, Lynch I, Ejtehadi MR, Monopoli MP, Bombelli FB, Laurent S. 2011. Protein-nanoparticle interactions: Opportunities and challenges. Chem Rev 111(9):5610-5637.

4. Knudsen SM, von Muhlen MG, Manalis SR. 2012. Quantifying particle coatings using high-precision mass measurements. Anal Chem 84(3):1240-1242.

5. Gagner JE, Lopez MD, Dordick JS, Siegel RW. 2011. Effect of gold nanoparticle morphology on adsorbed protein structure and function. Biomaterials 32(29):7241-7252.

6. Montes-Burgos I, Walczyk D, Hole P, Smith J, Lynch I, Dawson K. 2010. Characterisation of nanoparticle size and state prior to nanotoxicological studies. J Nanopart Res 12(1):47-53.

7. Walkey CD, Olsen JB, Guo HB, Emili A, Chan WCW. 2012. Nanoparticle size and surface chemistry determine serum protein adsorption and macrophage uptake. J Am Chem Soc 134(4):2139-2147.

8. Nejadnik MR, Deepak FL, Garcia CD. 2011. Adsorption of glucose oxidase to 3-D scaffolds of carbon nanotubes: Analytical applications. Electroanal 23(6):1462-1469.

9. Burg TP, Godin M, Knudsen SM, Shen W, Carlson G, Foster JS, Babcock K, Manalis SR. 2007. Weighing of biomolecules, single cells and single nanoparticles in fluid. Nature 446(7139):1066-1069.

10. Olcum S, Cermak N, Wasserman SC, Christine KS, Atsumi H, Payer KR, Shen WJ, Lee JC, Belcher AM, Bhatia SN, Manalis SR. 2014. Weighing nanoparticles in solution at the attogram scale. P Natl Acad Sci USA 111(4):1310-1315.

11. Patel AR, Lau D, Liu J. 2012. Quantification and characterization of micrometer and submicrometer subvisible particles in protein therapeutics by use of a suspended microchannel resonator. Anal Chem 84(15):6833-6840.

12. Weinbuch D, Zölls S, Wiggenhorn M, Friess W, Winter G, Jiskoot W, Hawe A. 2013. Micro-flow imaging and resonant mass measurement (archimedes)—complementary methods to quantitatively differentiate protein particles and silicone oil droplets. J Pharm Sci 102(7):21522165 .

13. von Muhlen MG, Brault ND, Knudsen SM, Jiang SY, Manalis SR. 2010. Label-free biomarker sensing in undiluted serum with suspended microchannel resonators. Anal Chem 82(5):19051910.

14. Norde W. 2008. My voyage of discovery to proteins in flatland ... And beyond. Colloid Surface B Biointerfaces 61(1):1-9.

15. Baier G, Costa C, Zeller A, Baumann D, Sayer C, Araujo PHH, Mailander V, Musyanovych A, Landfester K. 2011. BSA adsorption on differently charged polystyrene nanoparticles using isothermal titration calorimetry and the influence on cellular uptake. Macromol Biosci 11(5):628-638.

16. Lu HL, Lin DQ, Zhu MM, Yao SJ. 2012. Protein adsorption on DEAE ion-exchange resins with different ligand densities and pore sizes. J Sep Sci 35(22):3084-3090.

17. Bee JS, Chiu D, Sawicki S, Stevenson JL, Chatterjee K, Freund E, Carpenter JF, Randolph TW. 2009. Monoclonal antibody interactions with micro- and nanoparticles: Adsorption, aggregation, and accelerated stress studies. J Pharm Sci 98(9):3218-3238.

18. Feder J. 1980. Random sequential adsorption. J Theor Biol 87(2):237-254.

19. Van Beers MMC, Gilli F, Schellekens H, Randolph TW, Jiskoot W. 2012. Immunogenicity of recombinant human interferon beta 
interacting with particles of glass, metal, and polystyrene. J Pharm Sci 101(1):187-199.

20. Felhofer JL, Caranto JD, Garcia CD. 2010. Adsorption kinetics of catalase to thin films of carbon nanotubes. Langmuir 26(22):1717817183.

21. Roach P, Farrar D, Perry CC. 2005. Interpretation of protein adsorption: Surface-induced conformational changes. J Am Chem Soc 127(22):8168-8173.

22. van der Veen M, Stuart MC, Norde W. 2007. Spreading of proteins and its effect on adsorption and desorption kinetics. Colloid Surface B Biointerfaces 54(2):136-142.

23. Lee KB, Park SJ, Mirkin CA, Smith JC, Mrksich M. 2002. Protein nanoarrays generated by dip-pen nanolithography. Science 295(5560):1702-1705.

24. Vikholm I, Viitala T, Albers WM, Peltonen J. 1999. Highly efficient immobilisation of antibody fragments to functionalised lipid monolayers. Bba-Biomembranes 1421(1):39-52.

25. Monopoli MP, Walczyk D, Campbell A, Elia G, Lynch I, Bombelli FB, Dawson KA. 2011. Physical-chemical aspects of protein corona:
Relevance to in vitro and in vivo biological impacts of nanoparticles. $\mathrm{J}$ Am Chem Soc 133(8):2525-2534.

26. Casals E, Pfaller T, Duschl A, Oostingh GJ, Puntes V. 2010. Time evolution of the nanoparticle protein corona. ACS Nano 4(7):36233632 .

27. Monopoli MP, Aberg C, Salvati A, Dawson KA. 2012. Biomolecular coronas provide the biological identity of nanosized materials. Nat Nanotechnol 7(12):779-786.

28. Salvati A, Pitek AS, Monopoli MP, Prapainop K, Bombelli FB , Hristov DR, Kelly PM, Aberg C, Mahon E, Dawson KA. 2013. Transferrin-functionalized nanoparticles lose their targeting capabilities when a biomolecule corona adsorbs on the surface. Nat Nanotechnol 8(2):137-143.

29. Pan Y, Neuss S, Leifert A, Fischler M, Wen F, Simon U, Schmid G, Brandau W, Jahnen-Dechent W. 2007. Size-dependent cytotoxicity of gold nanoparticles. Small 3(11):1941-1949.

30. Jiang W, Kim BYS, Rutka JT, Chan WCW. 2008. Nanoparticlemediated cellular response is size-dependent. Nat Nanotechnol 3(3):145-150. 